\title{
BMJ Open Resilience and associative stigma among mental health professionals in a tertiary psychiatric hospital: a cross-sectional study in Singapore
}

Sherilyn Chang (D) , Louisa Picco, Edimansyah Abdin, Qi Yuan, Siow Ann Chong, Mythily Subramaniam

To cite: Chang S, Picco L, Abdin E, et al. Resilience and associative stigma among mental health professionals in a tertiary psychiatric hospital: a cross-sectional study in Singapore. BMJ Open 2019;9:e033762. doi:10.1136/ bmjopen-2019-033762

- Prepublication history and additional material for this paper are available online. To view these files, please visit the journal online (http://dx.doi org/10.1136/bmjopen-2019033762).

Received 21 August 2019 Revised 24 November 2019 Accepted 05 December 2019

Check for updates

(C) Author(s) (or their employer(s)) 2019. Re-use permitted under CC BY-NC. No commercial re-use. See rights and permissions. Published by BMJ.

Research Division, Institute of Mental Health, Singapore, Singapore

\section{Correspondence to} Sherilyn Chang; sherilyn_sh_chang@imh.com. $\mathrm{sg}$

\section{ABSTRACT}

Objectives The mental health profession exposes healthcare workers to unique stressors such as associative stigma (stigmatisation that is extended from the stigmatised patients to psychiatric professionals and is based on affiliation with an individual with mental illness). Enhancing resilience, or the ability to 'bounce back' from adversity, is found to be useful in reducing occupational stress and its negative effects. In view of the high burnout rates reported among mental health professionals, this study aimed to examine resilience in this group of professionals and to explore the association between resilience and associative stigma.

Design Observational study-cross-sectional design. Setting Tertiary psychiatry hospital in Singapore. Participants The study was conducted among 470 mental health professionals (doctors, nurses and allied health professionals) working in the hospital.

Measures Resilience was assessed using the Brief Resilience Scale (BRS) and participants completed questionnaires that examined associative stigma. Participants provided their sociodemographic information, length of service, and information on whether they knew of a close friend or family member who had a mental illness.

Results Mean resilience score for the overall sample was 3.59 ( $\mathrm{SD}=0.64)$. Older age $(\beta=0.012,95 \% \mathrm{Cl} 0.004$ to $0.019, p=0.003$ ) and having known a family member or close friend with a mental illness $(\beta=0.155,95 \% \mathrm{Cl}$ 0.019 to $0.290, p=0.025$ ) predicted higher BRS score. Associative stigma remained significantly associated with resilience score after controlling for sociodemographic factors whereby higher associative stigma predicted lower resilience scores.

Conclusion The present finding suggests that resilience building programmes among mental health workers should target those of the younger age group, and that addressing the issue of associative stigma is essential.

\section{INTRODUCTION}

Work in mental healthcare setting is fraught with stressors that are unique to this field. ${ }^{1}$ The nature of providing mental health services necessitates regular emotional and empathetic engagement with patients and their family members, and also occasional dealing
Strengths and limitations of this study

- One of the few studies that have examined resilience among mental health professionals and the association between resilience and associative stigma.

- Relatively large sample size with data collected from professionals across various occupational roles strengthens the generalisability of findings.

- Cross-sectional nature of the study, however, limits the ability to draw conclusions on causal effects.

with difficult and challenging behaviours of patients. ${ }^{2}$ Prolonged exposure to such workplace stressors has an impact on the wellbeing of mental health professionals ${ }^{34}$ and also compromises on their ability to provide quality care for patients. ${ }^{5}$

Stigma is a unique stressor for the field of mental healthcare. ${ }^{1}$ Negative stereotypes of and prejudice against mental health professionals might develop as a result of their close relationship with mental health patients. This is often termed 'associative stigma' wherein stigmatisation is extended from the stigmatised patients to psychiatric professionals ${ }^{6}$ and is based on affiliation with an individual with mental illness. ${ }^{7}$ Such stigmatisation is common among mental health professionals $^{689}$ and is associated with more depersonalisation, higher emotional exhaustion and poorer job satisfaction, ${ }^{8}$ and these associations were found to be significant even in longitudinal analyses. ${ }^{10}$ Studies have found that stigmatisation affects self-esteem ${ }^{11}$ and professional identities of psychiatric nurses ${ }^{12}$ and has bearing on career decisions and workplace retention. ${ }^{1314}$

There is an increased interest toward the construct of resilience as a positive trait that can buffer against the negative effects of such occupational stress. ${ }^{15}$ Resilience or the 'ability to bounce back or recover from stress ${ }^{16}$ is an 
important trait for professionals working in the mental health setting given the unique work stressors they face. Studies have found that having higher levels of resilience predicted lower psychological distress among mental health professionals. ${ }^{17} 18$ Additionally, individual and contextual factors contribute to resilience building in individuals. ${ }^{19}{ }^{20}$ In a literature review of resilience among health professionals, several factors that promoted resilience were identified, including maintaining a work-life balance, having a professional identity and having social support from family and friends. ${ }^{19}$

Several studies have looked at the association between stigma and resilience. In a qualitative study by Crowe $e t$ $a l$, the relationship between stigma and resilience was described as bidirectional: having resilience decreases the stigma experienced, while being stigmatised decreases one's resilience. ${ }^{21}$ Resilience serves to counter the stigma experienced, and at the same time stigma impinges on the ability to develop resilience. Additionally, stigmatised individuals felt obliged to build on their resilience through social support and enhance their personal attribute, such as positive emotions and strengths, to counteract the stigma. ${ }^{22}$ Resilience was found to be a coping technique endorsed by mental health professionals to manage associative stigma experiences. ${ }^{9}$

In view of the high level of stress and burnout that has been reported among mental health professionals in Singapore, ${ }^{23}$ it is important to better understand resilience among this group of professionals. There has only been one study which explored resilience among healthcare professionals in public hospitals in Singapore. ${ }^{24}$ This study found that more than $70 \%$ of the staff surveyed had encounters of workplace violence, and less than half of them performed positively on a resilience assessment. Staff who had mental health training were twice as likely to be resilient than those who did not attend such trainings. Though the study did not indicate the type of mental health trainings that staff had attended, the authors noted that these can range from mental health-related talks to certified therapy programmes that are aimed at enhancing 'resistance, resiliency and recovery of healthcare workers affected by personal or workplace stress or critical incidents'. In view of their findings, the authors recommended the provision of mental health and crisis intervention training for hospital staff to enhance their emotional resilience. Furthermore, mental health work is not viewed favourably by the public in Singapore; a study found that $67 \%$ of psychiatrists and $58 \%$ of psychiatric nurses surveyed had reported being laughed at for working with psychiatric patients. ${ }^{25}$ Approximately 30\% of them were discouraged by their family from engaging in this line of work. Interestingly, a U-shaped relationship has been proposed to describe the association between public stigma and familiarity; service providers may have higher public stigma toward mental illness owing to burden and associative stigma that comes alongside greater familiarity. ${ }^{26}$ However, while psychiatric professionals may be familiar with mental illness on the basis of their job, it is also possible that their familiarity stems from having personal contact with a friend or family member with mental health problems. Contact in a personal capacity may be different from that in a professional capacity in terms of evoking empathy or resilience when interacting with a family or friend with mental illness. It is hence important to account for any potential impact of having personal contact with an individual with mental illness when exploring the relationship of associative stigma with other concepts. Given the considerable stigma surrounding mental illness and mental health work in Singapore ${ }^{25} 27$ which may extend to mental healthcare providers and act as a workplace stressor, it is thus important to also examine the association between associative stigma and resilience among mental health professionals. The present study therefore aimed to examine correlates of resilience and its association with associative stigma among mental health professionals working at the Institute of Mental Health (IMH), which is the only tertiary psychiatric hospital in Singapore.

\section{METHODS \\ Study sample}

In this cross-sectional study, participants were recruited using convenience sampling. Emails inviting participation in the research study were sent out to mental health professionals including doctors, nurses and allied health staff (psychologists, pharmacists, occupational therapists, physiotherapists, case managers and medical social workers) working at IMH. A web link was provided in the email for staff to access the online survey which was administered using Questionpro, an online survey software. To be eligible, participants had to be aged 21 years and above and able to complete the online survey in English. Informed consent was obtained from the participants through the online portal and they were reimbursed on completion of the survey. A total of 470 mental health professionals were recruited for the study between February and April 2016. The study was approved by the National Healthcare Group Domain Specific Review Board in Singapore.

\section{Instruments}

Resilience was measured using the Brief Resilience Scale (BRS), a 6-item instrument that assesses the ability of individuals to bounce back or recover from stress. ${ }^{16}$ Participants indicated the extent to which they agree with each statement on a 5 -point scale ( $1=$ 'strongly disagree' to $5=$ 'strongly agree'). Examples of the items include 'I tend to bounce back quickly after hard times' and 'I usually come through difficult times with little trouble'. Negatively worded statements were first reversed coded and a BRS score was derived from the mean of the six items. The scale was found to have a one-factor structure and had obtained good internal consistency $(\alpha=0.80-0.91)$ and testretest reliability $(\mathrm{r}=0.62-0.69)$ in its validation study. ${ }^{16} \mathrm{It}$ 
demonstrated an acceptable level of internal consistency in the current study sample, with a Cronbach's alpha of 0.76 .

To examine associative stigma, measures of the construct were adapted from other studies ${ }^{78}$ and additional items were included based on our literature review as at the time the study was conducted, there was no standardised and well-validated associative stigma instrument available (see online supplementary appendix for the full list of items). For five of the items, participants responded to statements, such as 'People react negatively when they know I work in a mental care setting' and 'I feel ashamed to be working in a mental healthcare setting' using a 5-point scale ranging from 'never' to 'all the time'. ${ }^{8}$ For six additional items including 'Most people think less of a person who works in a mental healthcare setting' and 'Once they know a person works in a mental healthcare setting, most people will take their opinions less seriously', ${ }^{7}$ participants rated their level of agreement with these statements on a 5-point scale from 'strongly agree' to 'strongly disagree'. Among the same study sample, latent class analysis was conducted to classify underlying responses of associative stigma into mutually exclusive latent classes. ${ }^{28}$ Results revealed a threeclass model showed the best fit and comprised no/low, moderate and high associative stigma. These latent classes were used in the subsequent analysis. Detailed description of the findings for the latent class analysis has been reported elsewhere. ${ }^{28}$

Sociodemographic information including age, gender, ethnicity, marital status, educational attainment and residential status was collected. Participants provided information on whether they knew a close friend or family member who had a mental illness. Their occupation and length of service at IMH were also recorded.

\section{Statistical analysis}

Statistical analyses were carried out using Statistical Package for Social Sciences (SPSS) V.23. Descriptive statistics were used to describe the sample characteristics where mean and SD were calculated for continuous variables, and frequencies and percentages were calculated for categorical variables. One-way analysis of variance (ANOVA) test with post-hoc Bonferroni correction was conducted to examine group differences in BRS mean scores by occupational group. Multiple linear regression was used to explore sociodemographic correlates of resilience. BRS mean scores were entered as the outcome variable in the regression model and predictors were sociodemographic variables including age, gender, ethnicity, marital status, educational attainment, residential status, occupation, length of service and whether they knew a family member or close friend who had a mental illness. Next, the association between resilience and associative stigma was examined at both univariate and multivariate levels using linear regression model, where resilience scores were treated as the outcome variable and associative stigma as the predictor with and without controlling for sociodemographic variables. All statistically significant results were set at p-value $\leq 0.05$.
Table 1 Sociodemographic characteristics of study sample $(n=462)$

\begin{tabular}{|c|c|c|c|}
\hline & & Mean & SD \\
\hline \multirow[t]{2}{*}{ Age } & & 36.4 & 10.6 \\
\hline & & $\mathbf{n}$ & $\%$ \\
\hline \multirow[t]{2}{*}{ Gender } & Female & 291 & 63.0 \\
\hline & Male & 171 & 37.0 \\
\hline \multirow[t]{6}{*}{ Ethnicity } & Chinese & 278 & 60.2 \\
\hline & Malay & 36 & 7.8 \\
\hline & Indian & 64 & 13.9 \\
\hline & Filipino & 59 & 12.8 \\
\hline & Myanmar & 16 & 3.5 \\
\hline & Others & 9 & 2.0 \\
\hline \multirow[t]{2}{*}{ Marital status } & Never married & 205 & 44.4 \\
\hline & Ever married & 257 & 55.6 \\
\hline \multirow[t]{4}{*}{$\begin{array}{l}\text { Educational } \\
\text { attainment }\end{array}$} & $\begin{array}{l}\text { Secondary/‘O/N’ } \\
\text { Level }^{\star}\end{array}$ & 18 & 3.9 \\
\hline & 'A' Level†/diploma & 49 & 10.6 \\
\hline & Bachelor & 241 & 52.2 \\
\hline & Master and above & 154 & 33.3 \\
\hline \multirow{3}{*}{$\begin{array}{l}\text { Residential } \\
\text { status }\end{array}$} & Singapore citizen & 320 & 69.3 \\
\hline & Permanent resident & 59 & 12.8 \\
\hline & Non-resident & 83 & 18.0 \\
\hline \multirow[t]{3}{*}{ Occupation } & Doctor & 58 & 12.6 \\
\hline & Nurse & 201 & 43.5 \\
\hline & Allied health & 203 & 43.9 \\
\hline \multirow{4}{*}{$\begin{array}{l}\text { Service duration } \\
\text { in IMH }\end{array}$} & $<1$ year & 52 & 11.3 \\
\hline & $1-5$ years & 195 & 42.2 \\
\hline & $6-10$ years & 103 & 22.3 \\
\hline & $>10$ years & 112 & 24.2 \\
\hline \multirow{2}{*}{$\begin{array}{l}\text { Family/ } \\
\text { close friends } \\
\text { diagnosed with } \\
\text { mental illness }\end{array}$} & Yes & 130 & 28.1 \\
\hline & No & 332 & 71.9 \\
\hline
\end{tabular}

*Singapore-Cambridge GCE O-Level and N-Level examinations taken after 4 years of secondary school education.

†Singapore-Cambridge GCE A-Level examination taken on completion of preuniversity education.

GCE, General Certificate of Education; IMH, Institute of Mental Health.

\section{RESULTS}

Eight cases were removed from the analysis due to pattern responses being detected or participants not fulfilling the inclusion criteria. Analysis was conducted on the remaining 462 cases, and table 1 presents the characteristics of the study sample. The majority of the participants were female $(63.0 \%)$, Chinese $(60.2 \%)$, Singapore citizens $(69.3 \%)$ and had been working at IMH for $1-5$ years $(42.2 \%)$. Participants of this study were generally well educated, with the majority having a bachelor degree $(52.2 \%)$. There were 58 doctors, 201 nurses and 203 
Table 2 Resilience scores of overall sample and by occupation

\begin{tabular}{llll} 
& Mean & SD & P value \\
\hline Overall & 3.59 & 0.64 & $<0.001$ \\
Doctors & 3.81 & 0.62 & \\
Nurses & 3.46 & 0.61 & \\
Allied health & 3.65 & 0.65 & \\
\hline
\end{tabular}

Results in bold indicate statistical significance at $p$-value $\leq 0.05$.

allied health staff in this sample. Among the allied health professionals recruited, there were 57 case managers, 47 medical social workers, 43 psychologists, 28 pharmacists, 25 occupational therapists and 3 physiotherapists.
Table 2 presents the mean resilience score for the overall sample and by occupation. The mean score for the overall sample was $3.59(\mathrm{SD}=0.64)$, and statistically significant group differences were observed from the one-way ANOVA results $(\mathrm{F}(2,452)=8.681, \mathrm{p}<0.001)$. Post-hoc test using Bonferroni correction found that the mean score of nurses was significantly lower than that of doctors $(\mathrm{p}=0.001)$ and allied health staff $(\mathrm{p}=0.009)$. Results from the multiple linear regression showed that age was significantly associated with resilience, where older age predicted higher resilience scores (table 3; $\beta=0.012$, $\mathrm{p}=0.003$ ). Additionally, those who had a family member or close friend with a mental illness had significantly higher resilience scores $(\beta=0.155, \mathrm{p}=0.025)$ compared with those who did not.

Table 3 Correlates of resilience score

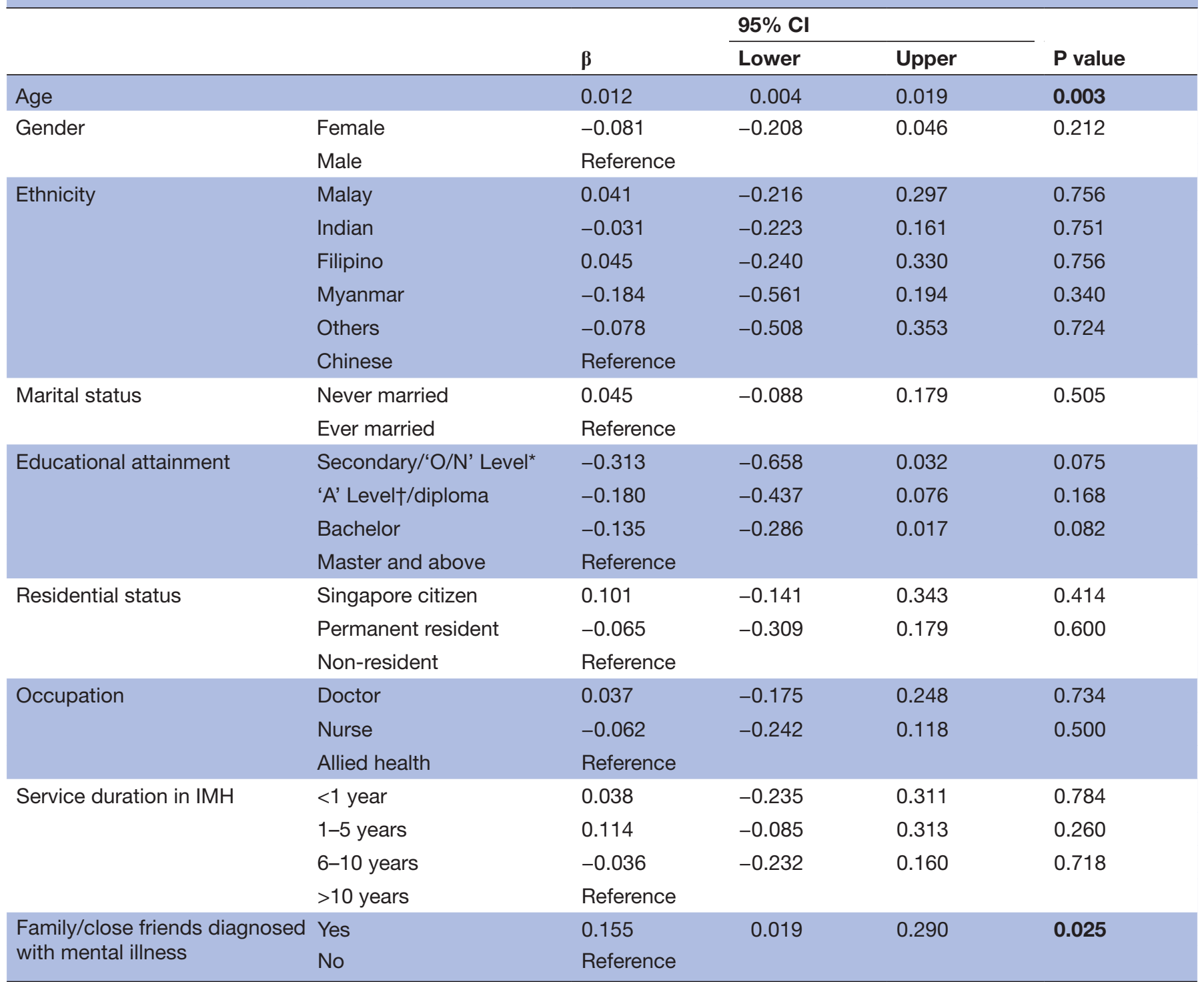

Results in bold indicate statistical significance at $p$-value $\leq 0.05$.

*Singapore-Cambridge GCE O-Level and N-Level examinations taken after 4 years of secondary school education. †Singapore-Cambridge GCE A-Level examination taken on completion of preuniversity education.

GCE, General Certificate of Education; IMH, Institute of Mental Health. 
Table 4 Resilience scores predicted by associative stigma class in adjusted and unadjusted regression model

\begin{tabular}{|c|c|c|c|c|}
\hline & \multirow[b]{2}{*}{$\boldsymbol{\beta}$} & \multicolumn{2}{|l|}{$95 \% \mathrm{Cl}$} & \multirow[b]{2}{*}{$P$ value } \\
\hline & & Lower & Upper & \\
\hline \multicolumn{5}{|l|}{ Unadjusted model } \\
\hline Moderate associative stigma & -0.265 & -0.384 & -0.146 & $<0.001$ \\
\hline High associative stigma & -0.590 & -0.776 & -0.404 & $<0.001$ \\
\hline No/low associative stigma & Reference & & & \\
\hline Moderate associative stigma & -0.271 & -0.390 & -0.151 & $<0.001$ \\
\hline High associative stigma & -0.577 & -0.767 & -0.386 & $<0.001$ \\
\hline
\end{tabular}

Results in bold indicate statistical significance at $p$-value $\leq 0.05$.

${ }^{*} \beta$ coefficient was derived from multiple linear regression after adjusted for age, gender, ethnicity, marital status, education, residential status, occupation, duration of service and if they know of a family/close friend diagnosed with mental illness.

Mean resilience scores for no/low, moderate and high associative stigma groups were 3.76, 3.49 and 3.17, respectively. Resilience was significantly associated with associative stigma at both the univariate and multivariate levels (table 4). After adjusting for potential confounding variables, resilience remained significantly associated with associative stigma. Participants who experienced moderate $(\beta=-0.271, \quad \mathrm{p}<0.001)$ and high associative stigma $(\beta=-0.577, p<0.001)$ had lower resilience scores than those with no/low associative stigma.

\section{DISCUSSION}

The present study aimed to examine resilience and its association with associative stigma among mental health professionals. A positive correlation was found between age and resilience with resilience increasing with age. Mental health professionals who personally knew of someone, a family member or close friend, diagnosed with mental illness also had a higher resilience score. Another main finding in this study was that individuals who experienced moderate and high levels of associative stigma had lower resilience scores.

Mental health professionals in this sample reported a moderate level of resilience with a sample mean of 3.59 . To the best of our knowledge, no other study has assessed resilience among psychiatric staff using the BRS. This limits the ability to draw any definite conclusion with regards to the level of resilience in our study sample. A study on first-year paediatric and medicine-paediatric residents reported a sample mean of $3.80,{ }^{29}$ while another study on young health professionals and trainees reported a mean of 3.60 using the BRS. ${ }^{30}$ These figures are higher than the mean obtained from this study, suggesting that mental health professionals have lower resilience. However, the aforementioned studies were conducted in the western context and the lower resilience score may instead reflect cultural differences in the notion of resilience. ${ }^{31}$ It is important to bear in mind the cultural dimensions when interpreting this difference. There are components of resilience that were found to be unique to eastern culture-religious faith and psychosocial gratitude $^{32}$ — which may not be adequately reflected in the current study as the questionnaire does not include these domains. It is plausible that individuals with an eastern sociocultural background may score 'higher' when resilience is defined in these aspects. More research is, therefore, required among mental health professionals to obtain comparable data and to investigate possible cultural differences of resilience.

The positive association found between age and resilience is not a surprising finding. In a study that looked at psychological resilience, older adults reported greater resilience than younger adults in the domains of emotional regulation and problem solving, though younger adults had greater resilience that was related to social support. ${ }^{33}$ With age comes a greater range of life experiences, through which individuals are more likely to have gained effective coping strategies and acquired useful resources that benefit their appraisal of stress, and hence building on their resilience. Given that the association between age and resilience found in the present study took into account the years of service at the hospital, it is likely that these resources extend beyond those which mental health professionals acquired within the organisation to those available from their immediate surrounding, for example, friends and family members.

Furthermore, having known someone who was diagnosed with mental illness was associated with higher resilience scores. It is plausible that having been on the recovery journey together with a friend or family with mental illness provided resources and skill set for this group of mental health professionals, in a way psychologically preparing them for whatever difficult times that may come. In times of adversity, they would be prepared to deal with the situation and able to 'bounce back' from the hardships.

Doctors were found to have the highest mean resilience scores as compared with nursing staff and allied health 
professionals in this study (table 2). However, these differences were not significant when accounted for other sociodemographic variables (table 3 ). This suggests that there may be a common pathway through which mental health professionals develop resilience, ${ }^{19}$ possibly through institutional support or that the similarities in the nature of work require comparable levels of resilience.

In this study, mental health professionals who experienced moderate and high associative stigma were found to have lower resilience. Having higher associative stigma meant that these individuals were more likely to endorse items, such as 'The mental health profession lacks a scientific basis' and 'People react negatively when they know I work in a mental healthcare setting'. In view of these negative perceptions pertaining to their own occupation, it suggests that mental health professions with moderate and high associative stigma may not identify with their job and not find pride in the work they do. Several studies have pointed out the importance of professional identity in relation to building resilience among healthcare workers including nurses, psychologists and social workers. ${ }^{19} 3435$ It is plausible that the lack of professional identity among those with moderate and high associative stigma accounts for the lower level of resilience in them. What this implies is that when they are stigmatised by others for the work they do, given that they do not identify with their job and feel ashamed about it, it may be more difficult for them to overcome work stressors they encounter, and thus less able to recover from stress (ie, lower resilience).

Another way to interpret the association between higher associative stigma and lower resilience is through the idea of social support. ${ }^{36}$ Mental health professionals experiencing moderate and high associative stigma are unlikely to receive emotional support from those who stigmatised them, given the perpetuators of associative stigma are those who trivialise the work that mental health professionals do. Thus in times of stress, these mental health workers would have lesser resources to tap on to bounce back from adversity, as compared with those experiencing no/low associative stigma. Additionally, there is some evidence to suggest that social support may play a mediating role in the association between resilience and associative stigma. A study found that mental health patients with higher levels of social support had lower levels of internalised stigma, and this mediated the negative association between societal stigma and recovery. ${ }^{37}$ It can thus be hypothesised that having social support reduces the impact of stigma on resilience through the pathway of internalised stigma. It would be interesting to test this hypothesised relationship among mental health professionals in future studies. It must be acknowledged that the relationship between stigma and resilience may be bidirectional. ${ }^{21}$ Given that resilience has been used as a strategy to cope with associative stigma, ${ }^{9}$ it is also possible that mental health professionals with lower resilience were more likely to experience higher associative stigma. Having lower resilience could lead these professionals to be more sensitive and perceive being stigmatised more than those who had higher levels of resilience. These professionals might also, as a result of having lower resilience, have lesser ability to counter the stigma they experienced, and therefore perceived experiencing a higher level of associative stigma. Future longitudinal studies would be needed to parse out the bidirectional nature of resilience and associative stigma.

It has been recommended that resilience building should be incorporated into training programmes for healthcare professionals. ${ }^{38}$ Given the unique stressors that psychiatric professionals encounter, it would be of practical use to implement resilience programmes in this group as well, particularly in the workplace setting. ${ }^{39}$ Findings from this study would suggest that such programmes can be targeted at younger professionals as they have lower levels of resilience. Resilience programmes for mental health professionals may need to address the issue of associative stigma, given that those who experienced higher associative stigma had lower resilience. These programmes can seek to promote professional identity among staff, placing emphasis on the importance of their work and increasing public recognition of it, as efforts to counteract stigma and enhancing resilience. Such workplace programmes may also serve to mitigate the negative effects of associative stigma among mental health professionals on service users' satisfaction and self-stigma that has been identified in previous research. ${ }^{8}$ Knowing that stigmatised individuals take steps to draw support from others, ${ }^{21} 22$ resilience programmes may also need to be augmented with a supportive network from within the organisation that acts a resource for psychiatric staff.

Findings from this study should be interpreted in view of its limitations. The present study was cross-sectional in nature, thus causal relationship between resilience and associative stigma cannot be established. The study sample was based on convenient sampling from a single tertiary psychiatric hospital, and thus the findings may be specific to mental health professionals in this context. Differences among staff within the allied health group itself were not investigated and there could be variation in resilience and associative stigma that is related to their work tasks.

\section{CONCLUSIONS}

Resilience, or the ability to 'bounce back' or recover from stress, is an important trait for mental health professionals in view of challenges in this field of work such as associative stigma. To the best of our knowledge, the present study is one of the first studies that has conducted a quantitative assessment of resilience among mental health professionals and examined its association with associative stigma. The mental health profession should look at ways to enhance resilience among mental health professionals, and addressing the issue of associative stigma might be one such approach. 
Contributors SC interpreted the findings, wrote the draft manuscript and critically revised the article. LP conceived the study, wrote the protocol and provided inputs for the manuscript. EA performed the statistical analysis and assisted in the interpretation of the findings. QY and SAC critically reviewed the article and provided their inputs. MS reviewed the study protocol and critically reviewed the article. All authors have read and approved the final manuscript.

Funding This research is supported by the Singapore Ministry of Health's National Medical Research Council under the Centre Grant Programme (Grant No: NMRC/ CG/004/2013).

\section{Competing interests None declared.}

Patient and public involvement statement There was no patient or public involvement in the study design; however, staff at IMH will be informed of the study findings.

Patient consent for publication Not required.

Ethics approval Ethical approval was obtained from the Domain Specific Review Board of the National Healthcare Group, Singapore (DSRB No: 2016/00043).

Provenance and peer review Not commissioned; externally peer reviewed.

Data availability statement Data are not available for online access, however, readers who wish to gain access to the data can write to the senior author MS at mythily@imh.com.sg with their requests. Access can be granted subject to the institutional review board (IRB) and the research collaborative agreement guidelines. This is a requirement mandated for this research study by our IRB and funders.

Open access This is an open access article distributed in accordance with the Creative Commons Attribution Non Commercial (CC BY-NC 4.0) license, which permits others to distribute, remix, adapt, build upon this work non-commercially, and license their derivative works on different terms, provided the original work is properly cited, appropriate credit is given, any changes made indicated, and the use is non-commercial. See: http://creativecommons.org/licenses/by-nc/4.0/.

\section{ORCID iD}

Sherilyn Chang http://orcid.org/0000-0001-9716-4543

\section{REFERENCES}

1 Rössler W. Stress, burnout, and job dissatisfaction in mental health workers. Eur Arch Psychiatry Clin Neurosci 2012;262 Suppl 2:65-9.

2 Jenkins R, Elliott P, Stressors EP. Stressors, burnout and social support: nurses in acute mental health settings. J Adv Nurs 2004;48:622-31.

3 Braun M, Schönfeldt-Lecuona C, Freudenmann RW, et al Depression, burnout and effort-reward imbalance among psychiatrists. Psychother Psychosom 2010;79:326-7.

4 Volpe U, Luciano M, Palumbo C, et al. Risk of burnout among early career mental health professionals. J Psychiatr Ment Health Nurs 2014;21:774-81.

5 Hall LH, Johnson J, Watt I, et al. Healthcare staff wellbeing, burnout, and patient safety: a systematic review. PLoS One 2016;11:e0159015.

6 Catthoor K, Hutsebaut J, Schrijvers D, et al. Preliminary study of associative stigma among trainee psychiatrists in Flanders, Belgium. World J Psychiatry 2014;4:62-8.

7 Ben Natan M, Drori T, Hochman O. Associative stigma related to psychiatric nursing within the nursing profession. Arch Psychiatr Nurs 2015;29:388-92.

8 Verhaeghe M, Bracke P. Associative stigma among mental health professionals: implications for professional and service user wellbeing. J Health Soc Behav 2012;53:17-32.

9 Vayshenker BA, DeLuca J, Bustle T, et al. "As soon as people hear that word...": associative stigma among clinicians working with people with serious mental illness. J Public Ment Health 2018;17:20-8.

10 Yanos PT, DeLuca JS, Salyers MP, et al. Cross-Sectional and prospective correlates of associative stigma among mental health service providers. Psychiatr Rehabil J 2019.

11 Sadow D, Ryder M. Reducing stigmatizing attitudes held by future health professionals: the person is the message. Psychol Serv 2008;5:362-72

12 Sercu C, Ayala RA, Bracke P. How does stigma influence mental health nursing identities? an ethnographic study of the meaning of stigma for nursing role identities in two Belgian psychiatric hospitals. Int J Nurs Stud 2015;52:307-16.

13 Agyapong VIO, Osei A, Farren CK, et al. Factors influencing the career choice and retention of community mental health workers in Ghana. Hum Resour Health 2015;13:56.

14 Gaebel W, Zäske H, Zielasek J, et al. Stigmatization of psychiatrists and general practitioners: results of an international survey. Eur Arch Psychiatry Clin Neurosci 2015;265:189-97.

15 Hao S, Hong W, Xu H, et al. Relationship between resilience, stress and burnout among civil servants in Beijing, China: mediating and Moderating effect analysis. Pers Individ Dif 2015;83:65-71.

16 Smith BW, Dalen J, Wiggins K, et al. The Brief Resilience Scale: assessing the ability to bounce back. Int J Behav Med 2008;15:194-200.

17 Harker R, Pidgeon AM, Klaassen F, et al. Exploring resilience and mindfulness as preventative factors for psychological distress burnout and secondary traumatic stress among human service professionals. Work 2016;54:631-7.

18 Kinman G, Grant L. Exploring stress resilience in trainee social workers: the role of emotional and social competencies. Br J Soc Work 2011;41:261-75.

19 McCann CM, Beddoe E, McCormick K, et al. Resilience in the health professions: a review of recent literature. Int $J$ Wellbeing 2013;3:60-81.

20 Tusaie K, Dyer J. Resilience: a historical review of the construct. Holist Nurs Pract 2004;18:3-8. quiz 9-10.

21 Crowe A, Averett P, Glass JS. Mental illness stigma, psychological resilience, and help seeking: what are the relationships? Ment Health Prev 2016;4:63-8.

22 Boardman F, Griffiths F, Kokanovic R, et al. Resilience as a response to the stigma of depression: a mixed methods analysis. $J$ Affect Disord 2011;135:267-76.

23 Yang S, Meredith P, Khan A. Stress and burnout among healthcare professionals working in a mental health setting in Singapore. Asian $J$ Psychiatr 2015;15:15-20.

24 Chan AOM, Chan YH, Kee JPC. Exposure to crises and resiliency of health care workers in Singapore. Occup Med 2013;63:141-4.

25 Lai YM, Hong CP, Chee CY. Stigma of mental illness. Singapore Med $J$ 2001;42:111-4.

26 Corrigan PW, Nieweglowski K. How does familiarity impact the stigma of mental illness? Clin Psychol Rev 2019;70:40-50.

27 Subramaniam M, Abdin E, Picco L, et al. Stigma towards people with mental disorders and its components - a perspective from multiethnic Singapore. Epidemiol Psychiatr Sci 2017;26:371-82.

28 Picco L, Chang S, Abdin E, et al. Associative stigma among mental health professionals in Singapore: a cross-sectional study. BMJ Open 2019;9:e028179.

29 Olson K, Kemper KJ, Mahan JD. What factors promote resilience and protect against burnout in first-year pediatric and medicinepediatric residents? J Evid Based Complementary Altern Med 2015;20:192-8

30 Kemper KJ, Mo X, Khayat R. Are mindfulness and Self-Compassion associated with sleep and resilience in health professionals? J Altern Complement Med 2015;21:496-503.

31 Ungar M. Cultural dimensions of resilience among adults. Handbook of adult resilience. New York, NY, US: The Guilford Press, 2010: 404-23.

32 Fernando GA. Bloodied but unbowed: resilience examined in a South Asian community. Am J Orthopsychiatry 2012;82:367-75.

33 Gooding PA, Hurst A, Johnson J, et al. Psychological resilience in young and older adults. Int J Geriatr Psychiatry 2012;27:262-70.

34 Beddoe L, Davys A, Adamson C. Educating resilient practitioners. Social Work Education 2013:32:100-17.

35 Carson E, King S, Papatraianou LH. Resilience among social workers: the role of informal learning in the workplace. Practice 2011;23:267-78.

36 Son Hing LS. Responses to stigmatization: the Moderating roles of primary and secondary appraisals. Du Bois Review: Social Science Research on Race 2012;9:149-68.

37 Chronister J, Chou C-C, Liao H-Y. The role of stigma coping and social support in mediating the effect of societal stigma on internalized stigma, mental health recovery, and quality of life among people with serious mental illness. J Community Psychol 2013;41:582-600.

38 McAllister M, McKinnon J. The importance of teaching and learning resilience in the health disciplines: a critical review of the literature. Nurse Educ Today 2009;29:371-9.

39 Howard R, Kirkley C, Baylis N. Personal resilience in psychiatrists: systematic review. BJPsych Bull 2019;43:209-15. 